\title{
Increased mediator complex subunit 15 expression is associated with poor prognosis in hepatocellular carcinoma
}

\author{
KUNYUAN WANG, CHENXI DUAN, XUEJING ZOU, YANG SONG, WENWEN LI, \\ LUSHAN XIAO, JIE PENG, LIHENG YAO, QIAN LONG and LI LIU
}

\begin{abstract}
Hepatology Unit and Department of Infectious Diseases, Nanfang Hospital, Southern Medical University, Guangzhou, Guangdong 510515, P.R. China
\end{abstract}

Received April 8, 2017; Accepted November 10, 2017

DOI: $10.3892 / 01.2018 .7820$

\begin{abstract}
Mediator complex subunit 15 (MED15) is a coactivator involved in the regulated transcription of RNA polymerase II-dependent genes and serves an oncogenic role in numerous types of cancer. However, the expression and function of MED15 in hepatocellular carcinoma (HCC) remain unknown. In the present study, the aim was to investigate the expression and clinical significance of MED15 in HCC. Reverse transcription-quantitative polymerase chain reaction (RT-qPCR) and immunohistochemical analysis revealed that MED15 mRNA and protein levels were significantly upregulated in HCC tissues compared with those in the corresponding adjacent non-tumor liver tissues. Furthermore, analyzing data from The Cancer Genome Atlas-Liver Hepatocellular Carcinoma (TCGA-LIHC) and GSE14520 datasets revealed a significant correlation between MED15 expression and the tumor size $(\mathrm{P}=0.033)$, Barcelona Clinic Liver Cancer stage $(\mathrm{P}=0.031), \alpha$-fetoprotein levels $(\mathrm{P}=0.002)$ and metastasis risk $(\mathrm{P}=0.001)$. Furthermore, patients with high MED15 expression levels had a shorter survival time compared with those with low MED15 expression levels $(\mathrm{P}<0.05)$. Univariate and multivariate analyses further revealed that MED15 may be an independent prognostic factor for the overall survival of HCC patients (hazard ratio, 1.762; 95\% confidence interval, 1.077-2.882; $\mathrm{P}<0.05)$. In addition, MED15 expression was positively associated with hypoxia-inducible factor $1 \alpha$ expression in the TCGA-LIHC and GSE14520 datasets $(\mathrm{P}<0.01)$. In conclusion, the data reported in the present study indicated that MED15 is overexpressed in HCC and may represent a novel prognostic biomarker for patients with HCC.
\end{abstract}

Correspondence to: Professor Li Liu, Hepatology Unit and Department of Infectious Diseases, Nanfang Hospital, Southern Medical University, 1838 Guangzhou Avenue North Road, Guangzhou, Guangdong 510515, P.R. China

E-mail: liuli.fimmu@gmail.com

Key words: mediator complex subunit 15, hepatocellular carcinoma, upregulation, prognosis, hypoxia-inducible factor $1 \alpha$

\section{Introduction}

Hepatocellular carcinoma (HCC) is estimated to be the second most common cause of cancer-associated mortality worldwide, with steadily increasing morbidity and mortality rates $(1,2)$. Notably, approximately $50 \%$ of the liver cancer cases and mortalities worldwide occur in China (2). Although surgical resection and liver transplantation are regarded as sufficient treatment strategies to extend the life expectancy of certain patients with HCC (3), the prognosis of these patients remains unsatisfactory, since the disease is frequently diagnosed at an advanced stage and is typically accompanied by severe liver dysfunction. Therefore, there is an urgency to identify potential biomarkers for the early detection and prognostic prediction of HCC.

Mediator complex subunit 15 (MED15) is a subunit of the MED multiprotein complex and functions as a crucial cofactor for diverse signaling pathways, such as the sterol regulatory element-binding protein (SREBP) and transforming growth factor- $\beta$ signaling pathways (4-7). It has been reported that MED15 promotes transcriptional activation in eukaryotes and has been linked to certain human diseases (7-9). For instance, MED15 regulates the activity of the transcriptional activator SREBP and functions as a transcriptional cofactor to control lipid homeostasis in cardiovascular disease $(5,7)$. In addition, previous studies have demonstrated that dysregulation of MED15 expression promotes tumorigenesis. Increased MED15 expression has been reported in several human malignancies, including breast cancer, head and neck squamous cell carcinoma (HNSCC), prostate cancer and testicular germ cell tumors $(6,10-14)$. Furthermore, knockdown of MED15 significantly reduces the viability of cancer cells, indicating that disrupting MED15 expression may inhibit the progression of these types of cancer (10-12). However, the expression level of MED15 in HCC and its prognostic significance for clinical patients remain unknown.

In the present study, the aim was to investigate MED15 mRNA and protein expression levels in HCC tissues using reverse transcription-quantitative polymerase chain reaction (RT-qPCR) and immunohistochemical analysis, respectively. The study results revealed that MED15 levels were significantly upregulated in HCC tissues as compared with those in the corresponding adjacent non-tumor liver tissues. 
Additionally, analysis of The Cancer Genome Atlas-Liver Hepatocellular Carcinoma (TCGA-LIHC) and GSE14520 datasets revealed that MED15 expression levels were associated with hypoxia-inducible factor $1 \alpha$ (HIF-1 $\alpha$ ) expression and poor prognosis in patients with HCC. These findings suggest that MED15 serves a role in liver tumorigenesis and may be a potential therapeutic target for HCC treatment.

\section{Materials and methods}

Patients and tissue samples. The experiments of the present retrospective study were reviewed and approved by the Ethics Committee of Nanfang Hospital, Southern Medical University (Guangzhou, China). The enrolled patients had not received any anticancer therapies prior to surgery. All eligible patients provided informed consent prior to the collection of HCC specimens and corresponding adjacent non-tumor tissues. A total of 20 pairs of HCC tissues and matching adjacent non-tumor tissues were obtained from patients undergoing hepatectomy procedures between January 2014 and December 2015.

Each sample was divided into two parts, one part was immediately stored in RNA keeper tissue stabilizer (Vazyme Biotech Co., Ltd. Nanjing, China) and frozen at $-80^{\circ} \mathrm{C}$ prior to RNA extraction. The other was fixed overnight in $10 \%$ formaldehyde at room temperature and paraffin-embedded using conventional methods.

RNA extraction, $c D N A$ synthesis and $R T-q P C R$ analysis. Total RNA was extracted from the HCC tissues and paired adjacent non-tumor liver tissues using TRIzol $^{\circledR}$ reagent (Invitrogen; Thermo Fisher Scientific, Inc., Waltham, MA, USA), according to the manufacturer's protocol. RNAse-free DNase I (Takara Biotechnology Co., Ltd., Dalian, China) was used to remove genomic DNA contamination, while the quality and concentration of total RNA were measured using a NanoDrop ${ }^{\circledR}$ spectrophotometer (Thermo Fisher Scientific, Inc.). RNA was then transcribed into cDNA using an RT kit (Takara Biotechnology Co., Ltd.) in a $20-\mu 1$ reaction volume with $1 \mu \mathrm{g}$ RNA. Subsequently, amplification reactions were performed using a SYBR Green PCR kit from Takara Biotechnology Co., Ltd., using the following primers: MED15 sense, 5'-TGTCGTGTCTACGGCAACTC-3', and anti-sense, 5'-CACTCTGCTGAGCCTGGAA-3'; $\beta$-actin sense, 5'-TCA AGATCATTGCTCCTCCTGA-3', and anti-sense, 5'-CTC GTCATACTCCTGCTTGCTG-3'. $\beta$-actin was amplified as an internal control. Gene-specific amplification was performed using a LightCycler ${ }^{\circledR} 480$ Instrument II (Roche Diagnostics, Basel, Switzerland). Specific conditions for the qPCR reaction were as follows: Preliminary denaturation at $95^{\circ} \mathrm{C}$ for $30 \mathrm{sec}$, followed by 40 cycles of $95^{\circ} \mathrm{C}$ for $5 \mathrm{sec}$, and $60^{\circ} \mathrm{C}$ for $20 \mathrm{sec}$. A melting curve analysis of the PCR products was used to assess the specificity of amplification. Fold changes were calculated using the relative quantification $\left(2^{-\Delta \Delta \mathrm{Cq}}\right)$ method (15).

Hematoxylin and eosin staining. Human HCC tissues and matched adjacent non-tumor liver tissues were fixed in $10 \%$ formaldehyde for $24 \mathrm{~h}$, dehydrated through an ethanol series for $2 \mathrm{~h}$ each, followed by two washes in xylene (30 min each). After two immersions in paraffin (45 min each), tissues were embedded and sectioned. Tissue sections were incubated at $60^{\circ} \mathrm{C}$ for $1 \mathrm{~h}$ to remove the paraffin. Subsequently, they were stained with hematoxylin for $7 \mathrm{~min}$, de-stained in a hydrochloric acid alcohol solution for $15 \mathrm{sec}$ and then stained with eosin for $3 \mathrm{~min}$. Finally, sections were dehydrated through an ethanol series for $1 \mathrm{~min}$ each, rinsed with xylene and coverslipped with mounting medium. Staining results were observed and imaged under a light microscope (Olympus BX 51; Olympus Corporation, Tokyo, Japan).

Immunohistochemical assay. In total, 12 pairs of formalin-fixed and paraffin-embedded HCC tissues were used for MED15 immunohistochemical staining. Briefly, paraffin-embedded tissues were sliced into $4-\mu \mathrm{m}$ sections and incubated at $60^{\circ} \mathrm{C}$ for $2 \mathrm{~h}$. Following deparaffinization, the sections were treated with $3 \%$ hydrogen peroxide to inactivate the endogenous peroxidase, and antigens were retrieved using a citrate buffer. Next, the sections were incubated in PBS containing 5\% normal goat serum (cat. no. C-0005; BIOSS, Beijing, China) at room temperature for $1 \mathrm{~h}$, followed by incubation at $4^{\circ} \mathrm{C}$ overnight with a rabbit monoclonal anti-human MED15 antibody (1:200 dilution; ab181158; Abcam, Cambridge, UK) or with PBS alone, serving as the control. The following day, sections were rinsed three times with PBS and then incubated with a horseradish peroxidase-conjugated secondary antibody (Dako; Agilent Technologies, Inc., Santa Clara, CA, USA) at room temperature for $1 \mathrm{~h}$. Subsequent to rinsing with PBS, the sections were stained with 3,3'-diaminobenzidine (Dako; Agilent Technologies, Inc.) to induce a color reaction. The tissues were then counterstained with hematoxylin for $1 \mathrm{~min}$ and de-stained with acid-alcohol for $5 \mathrm{sec}$. Finally, the sections were dehydrated using an ethanol series (1 min each), rinsed in xylene, dried and placed on coverslips with a mounting medium. Immunohistochemical staining results were assessed under a light microscope at x200 and x400 magnification. MED15 positive staining was mainly present in the nuclei and manifested as a color varying between brown and light yellow. Compared with the background, the staining intensity was evaluated as follows: Light yellow, indicating weak expression; brownish-yellow, indicating moderate expression; and brown, indicating strong expression. MED15 protein expression in HCC was further analyzed using clinical specimens from The Human Protein Atlas (www.proteinatlas.org), which included 12 paired of HCC tissues and samples that can be filtered based on the level of antibody staining via selecting one or several of the following categories: High, medium, low and not detected.

TCGA-LIHC and GSE14520 datasets, Oncomine gene expression array. In order to investigate the role of MED15 expression in HCC, the current study analyzed the TCGA-LIHC dataset (http://www.cbioportal.org/data_sets.jsp) that contains 331 HCC cases and the GSE14520 dataset (https://www.ncbi .nlm.nih.gov/geo/query/acc.cgi?acc $=$ GSE14520) that provides 246 HCC cases (hereafter defined as the TCGA-LIHC and GSE14520 cohorts, respectively). These datasets comprise follow-up information for patients and gene expression data. Patients with available data on MED15 expression and follow-up information were included into the present study. Correlations of MED15 expression with the clinicopathological 
features, overall survival (OS) and disease-free survival (DFS) of patients with HCC were analyzed. Patients were organized according to their MED15 expression levels from low to high expression, and then divided into two groups. The first third of MED15 expression was defined as the MED15-low expression group and the remainder patients were termed the MED15-high expression group. The expression of MED15 in $\mathrm{HCC}$ was further analyzed on Oncomine ${ }^{\mathrm{TM}}$ gene expression array (https://www.oncomine.org/resource/login.html).

We used the OncoLnc online system (http://www.oncolnc .org/) to analyze the overall survival of HCC patients from the TCGA-LIHC dataset. According to the mRNA expression level of MED15 on the website, the first third of MED15 expression was defined as the MED15-low expression group and the remainder patients were termed the MED15-high expression group. And the result of patients' overall survival was presented on the website.

Gene set enrichment analysis (GSEA). The patients were divided into two groups according to the MED15 mRNA expression level in the TCGA-LIHC dataset, which were MED15 high- and low-expressed groups. Subsequently, GSEA software (GSEA Desktop Application v3.0; Broad Institute, Inc., Cambridge, MA, USA) was used to analyze, annotate and interpret enrichment results. Prior to running GSEA, four input files were prepared; the GCT, GMT, CHIP and cls formatted files. The GMT file was created in-house, and the GCT, CHIP and cls files were downloaded from the TCGA dataset. Gene sets that were positively or negatively correlated with the MED15 high- or low-expressed groups were searched for.

Statistical analysis. Student's t-test was used to analyze the RT-qPCR results, while Mann-Whitney U-test was used to evaluate the association between MED15 expression and the clinicopathological features of patients with HCC. OS and DFS time were calculated using the Kaplan-Meier method and analyzed using log-rank tests. Cox proportional hazards modeling was used for univariate and multivariate analysis to investigate the effect of variables on survival. Conventional clinicopathological variables and MED15 expression were enrolled into a Cox univariate regression analysis. Statistically significant variables from the univariate regression model were reanalyzed using the multivariate model. Statistical analyses were conducted using SPSS version 17.0 software (SPSS, Inc., Chicago, IL, USA). All statistical tests were two-sided, and $\mathrm{P}<0.05$ was considered to indicate a difference that was statistically significant.

\section{Results}

MED15 mRNA expression is elevated in HCC tissues. To investigate MED15 mRNA expression in HCC, 20 pairs of fresh HCC specimens and their corresponding adjacent non-tumor tissues were used to measure the relative expression of MED15 by RT-qPCR. It was observed that MED15 mRNA was notably increased in $\mathrm{HCC}$ tissues compared with that in non-tumor liver tissues (Fig. 1A and B). Next, to further validate the observed MED15 dysregulation in HCC, MED15 gene expression was analyzed in patients included in the TCGA-LIHC and GSE14520 datasets. In the unpaired TCGA-LIHC cohort, MED15 expression was overexpressed in HCC tissues compared with that in non-tumor liver tissues $(\mathrm{P}<0.001$; Fig. $1 \mathrm{C})$. In the paired TCGA-LIHC cohort, a similar result was observed when MED15 expression was re-assessed in 50 paired HCC samples and adjacent non-tumor liver samples ( $\mathrm{P}<0.001$; Fig. 1D). These findings were further confirmed by assessing MED15 expression in the GSE14520 dataset, which revealed that MED15 was upregulated in HCC tissues compared with that in non-tumor liver tissues from both unpaired and paired GSE14520 cohorts $(\mathrm{P}<0.05$; Fig. 1E and F). Furthermore, similar results were obtained by analyzing the expression data from Oncomine gene expression array datasets (data not shown). Collectively, these data indicated that MED15 mRNA expression is elevated in HCC tissues.

MED15 protein is overexpressed in human HCC specimens. Hematoxylin and eosin staining was conducted to distinguish HCC tissues from the corresponding adjacent non-tumor liver tissues (Fig. 2A). In addition, immunohistochemical analysis was used to detect the MED15 protein expression levels in human liver cancer tissues, and varying expression was observed. Positive immunostaining of MED15 appeared as granular brown-colored staining, which was mainly located in the nucleus of tumor cells (Fig. 2B). It was also observed that MED15 protein was highly expressed in the majority of HCC tissues as compared with that in the corresponding non-tumor tissues, and 9 out of the $12 \mathrm{HCC}$ samples investigated were positive for MED15 immunostaining.

To further confirm the aforementioned results, MED15 protein expression was analyzed in clinical specimens from The Human Protein Atlas. This analysis revealed that MED15 was positively and strongly expressed in HCC samples, whereas it was negatively or weakly expressed in normal hepatic tissues (Fig. 3A). Online immunohistochemical results obtained from The Human Protein Atlas revealed that MED15 protein was strongly expressed in 7 of the HCC tissues, moderately expressed in 4 specimens and weakly expressed in 1 sample (Fig. 3B and C). These findings indicated that MED15 protein is overexpressed in HCC.

Correlation between MED15 expression and clinicopathological characteristics of HCC. Since MED15 was upregulated in $\mathrm{HCC}$, the current study attempted to explore the potential oncogenic role of MED15 in HCC. Evidence of a correlation between MED15 expression and the clinicopathological status of patients with HCC was searched in the TCGA-LIHC and GSE14520 datasets. Statistical analysis of the TCGA-LIHC cohort indicated that MED15 expression was positively associated with the neoplasm grade $(\mathrm{P}=0.005)$ and $\alpha$-fetoprotein (AFP) levels ( $\mathrm{P}=0.001)$. However, no statistically significant correlation was observed between MED15 and other clinicopathological features (Table I). As summarized in Table II, significant correlations were also detected between MED15 expression and certain clinicopathological features in the GSE14520 cohort, including the tumor size $(\mathrm{P}=0.033)$, Barcelona Clinic Liver Cancer (BCLC) stage ( $\mathrm{P}=0.031)$, AFP levels $(\mathrm{P}=0.002)$ and metastasis risk $(\mathrm{P}=0.001)$. However, no statistically significant association was identified between MED15 expression and other clinicopathological characteristics, such as the patient sex, age, tumor number, TNM staging, cirrhosis and relapse. 
A
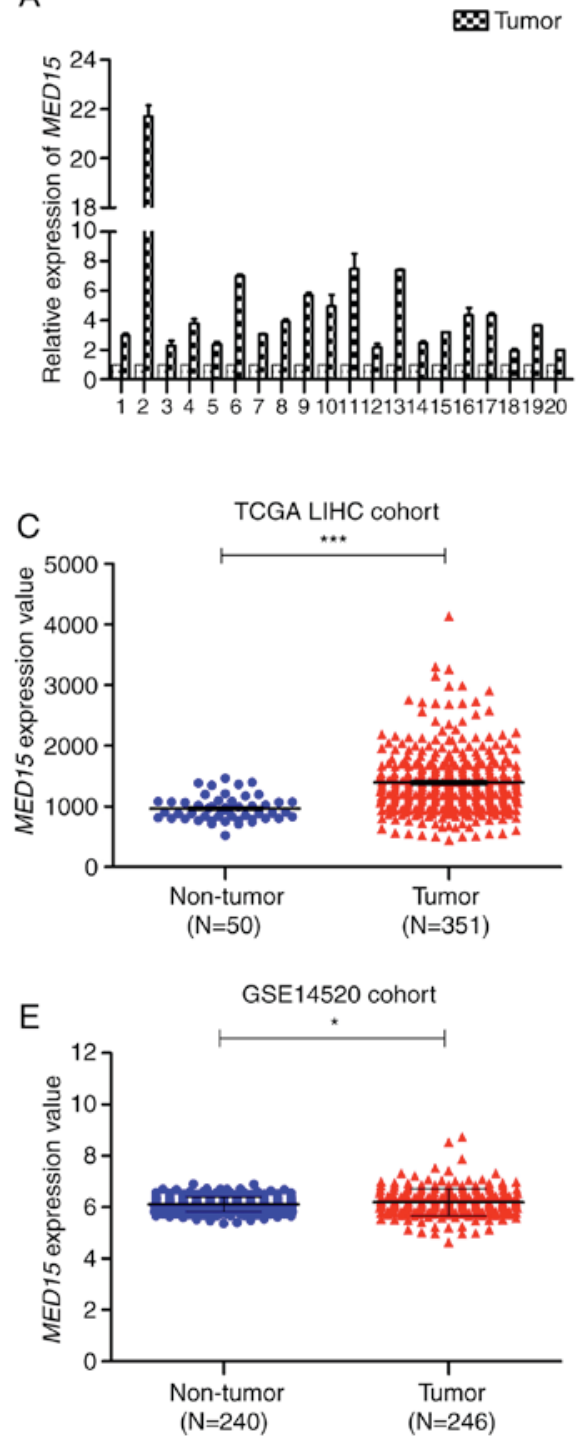
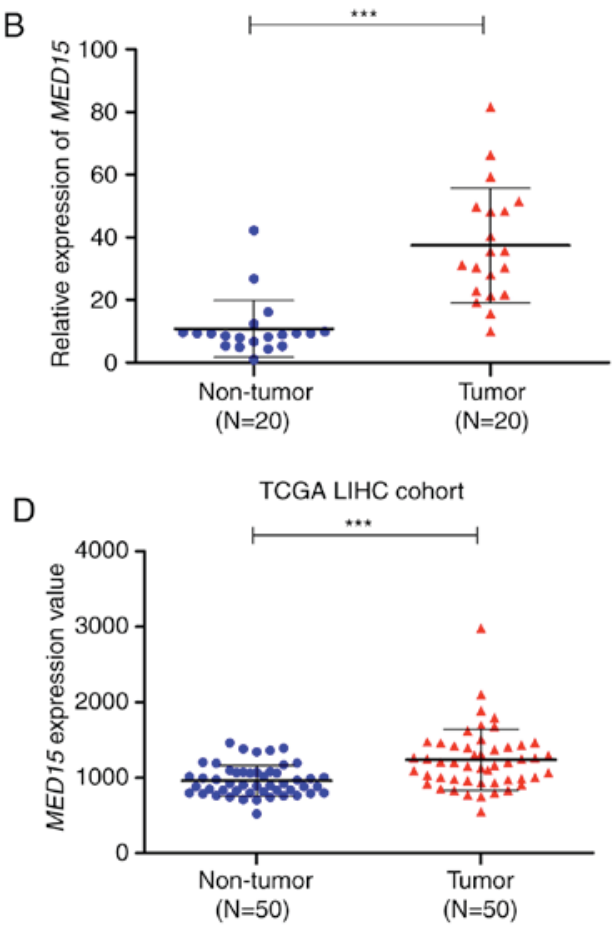

$\mathrm{F}$

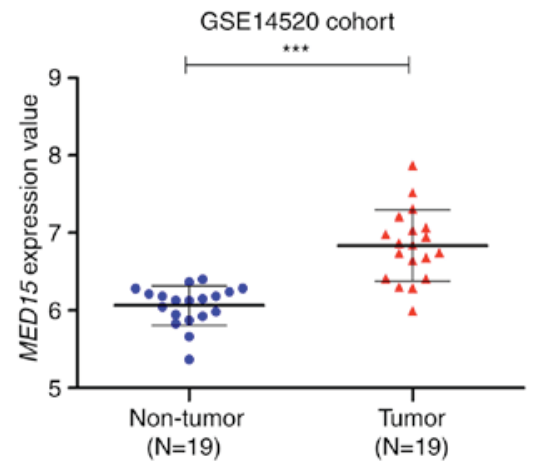

Figure 1. MED15 mRNA is upregulated in HCC tissues. (A) MED15 mRNA expression was analyzed by reverse transcription-quantitative polymerase chain reaction in 20 pairs of HCC tissues and matched adjacent non-tumor liver tissues. $\beta$-actin expression served as an internal control. (B) MED15 mRNA levels were significantly increased in 20 pairs of HCC specimens, as determined by a paired Student's t-test. Furthermore, MED15 mRNA expression was significantly higher in HCC tissues as compared with non-tumor tissues in the (C) unpaired ( $\mathrm{n}=331)$ and (D) paired ( $\mathrm{n}=50)$ TCGA-LIHC cohort, as well as in the (E) unpaired $(\mathrm{n}=246)$ and $(\mathrm{F})$ paired $(\mathrm{n}=19)$ GSE14520 cohort. ${ }^{*} \mathrm{P}<0.05$ and ${ }^{* * *} \mathrm{P}<0.001$. MED15, mediator complex subunit 15; HCC, hepatocellular carcinoma; TCGA-LIHC, The Cancer Genome Atlas-Liver Hepatocellular Carcinoma.

MED15 expression levels predict the clinical outcome of patients with HCC. Kaplan-Meier and log-rank test analyses were employed to test for an association between MED15 expression and the OS or DFS of the patients. The results on the TCGA-LIHC cohort data revealed that patients with highly expressed MED15 in tumor tissues exhibited a significantly shorter survival time as compared with those with low MED15 expression levels ( $\mathrm{P}=0.03$; Fig. 4A). This observation was supported by the OncoLnc online system (http://www .oncolnc.org/), which was used to explore links between TCGA-LIHC survival data and mRNA, miRNA or lncRNA expression levels, and revealed that MED15 expression levels were negatively associated with the $\mathrm{OS}(\mathrm{P}=0.028$; Fig. 4B). Furthermore, a comparative microarray analysis of gene expression was performed in the MED15-high and MED15-low expression groups using GSEA. This analysis revealed that tumor samples with high MED15 expression levels were enriched in the HCC survival reduction dataset and in the HCC recurrence increase dataset (Fig. 4C). However, analysis of the TCGA-LIHC cohort revealed no correlation between MED15 expression and DFS (Fig. 4D). Consistent with these findings, Kaplan-Meier and log-rank test analyses in the GSE14520 cohort demonstrated that high MED15 expression in tumor specimens was associated with a poorer outcome of patients $(\mathrm{P}=0.05$ for $\mathrm{OS}$ and $\mathrm{P}=0.11$ for DFS; Fig. 4E and F). According to these findings, it can be deduced that a high MED15 expression predicts a worse prognosis for patients with HCC.

MED15 may be a valuable prognostic marker for patients with $H C C$. Univariate and multivariate analyses were performed to estimate the prognostic variables in patients included in the 
Table I. Correlation between MED15 expression and hepatocellular carcinoma clinicopathological features in The Cancer Genome Atlas-Liver Hepatocellular Carcinoma cohort.

\begin{tabular}{|c|c|c|c|c|}
\hline \multirow[b]{2}{*}{ Variable } & \multirow[b]{2}{*}{ Total, $\mathrm{n}$} & \multicolumn{2}{|c|}{ MED15 expression, $\mathrm{n}$} & \multirow[b]{2}{*}{ P-value } \\
\hline & & High & Low & \\
\hline $\operatorname{Sex}$ & 331 & & & 0.584 \\
\hline Male & 219 & 140 & 79 & \\
\hline Female & 112 & 75 & 37 & \\
\hline Age (years) & 331 & & & 0.139 \\
\hline$\leq 50$ & 66 & 48 & 18 & \\
\hline$>50$ & 265 & 167 & 98 & \\
\hline Family cancer history & 288 & & & 0.106 \\
\hline Yes & 104 & 62 & 42 & \\
\hline No & 184 & 127 & 57 & \\
\hline Lymphocyte infiltration & 212 & & & 0.455 \\
\hline Absent & 106 & 59 & 47 & \\
\hline Mild & 91 & 60 & 31 & \\
\hline Severe & 15 & 7 & 8 & \\
\hline Vascular tumor cell invasion & 278 & & & 0.123 \\
\hline None & 183 & 109 & 74 & \\
\hline Micro & 82 & 52 & 30 & \\
\hline Macro & 13 & 12 & 1 & \\
\hline Neoplasm grade & 326 & & & $0.005^{\mathrm{a}}$ \\
\hline $\mathrm{G} 1+\mathrm{G} 2$ & 207 & 123 & 84 & \\
\hline $\mathrm{G} 3+\mathrm{G} 4$ & 119 & 89 & 30 & \\
\hline Pathologic stage & 310 & & & 0.544 \\
\hline $\mathrm{I}+\mathrm{II}$ & 226 & 145 & 81 & \\
\hline III+IV & 84 & 57 & 27 & \\
\hline AFP level (ng/ml) & 245 & & & $<0.001^{\mathrm{a}}$ \\
\hline$<400$ & 189 & 110 & 79 & \\
\hline$\geq 400$ & 56 & 47 & 9 & \\
\hline Child-Pugh classification grade & 208 & & & 0.658 \\
\hline A & 188 & 113 & 75 & \\
\hline $\mathrm{B}+\mathrm{C}$ & 20 & 11 & 9 & \\
\hline Liver fibrosis Ishak score & 191 & & & 0.891 \\
\hline$\leq 4$ & 123 & 70 & 53 & \\
\hline$>4$ & 68 & 38 & 30 & \\
\hline Relapse & 330 & & & 0.390 \\
\hline Yes & 133 & 83 & 50 & \\
\hline No & 197 & 132 & 65 & \\
\hline
\end{tabular}

${ }^{a}$ Value indicates a statistically significant difference. MED15, mediator complex subunit 15; AFP, $\alpha$-fetoprotein.

TCGA-LIHC and GSE14520 cohorts. In the TCGA-LIHC cohort, univariate analysis indicated that MED15 expression was significantly associated with the OS rate of patients with HCC ( $\mathrm{P}=0.032$; Table III). Multivariate analysis was then conducted using a Cox proportional hazards model and revealed that MED15 expression may be an independent prognostic factor for patients with HCC (hazard ratio (HR), 1.762; 95\% confidence interval $(\mathrm{CI}), 1.077-2.882 ; \mathrm{P}=0.024)$. While in the GSE14520 cohort, it was indicated that the number of tumors, tumor size, BCLC staging, TNM staging, AFP level, Cirrhosis, metastasis risk and relapse rather than MED15 expression were associated with OS rate of patients with HCC via univariate analysis (Table IV). Multivariate analysis demonstrated that the number of tumors (HR, 0.389; 95\% CI, 0.203-0.745; $\mathrm{P}=0.004)$, BCLC staging (HR, 3.615; 95\% CI, 1.982-6.593; $\mathrm{P}<0.001)$, metastasis risk (HR, 2.41; 95\% CI, 1.523-3.814; $\mathrm{P}<0.001)$, cirrhosis $(\mathrm{HR}, 4.456 ; 95 \% \mathrm{CI}$, 1.081-18.367; $\mathrm{P}=0.039)$, and relapse (HR, 91.516; 95\% CI, 
Table II. Correlation between MED15 expression and hepatocellular carcinoma clinicopathological features in the GSE14520 cohort.

\begin{tabular}{|c|c|c|c|c|}
\hline \multirow[b]{2}{*}{ Variable } & \multirow[b]{2}{*}{ Total, $\mathrm{n}$} & \multicolumn{2}{|c|}{ MED15 expression, $\mathrm{n}$} & \multirow[b]{2}{*}{ P-value } \\
\hline & & High & Low & \\
\hline Sex & 241 & & & 0.756 \\
\hline Male & 210 & 148 & 62 & \\
\hline Female & 31 & 21 & 10 & \\
\hline Age (years) & 241 & & & 0.768 \\
\hline$\leq 50$ & 124 & 88 & 36 & \\
\hline$>50$ & 117 & 81 & 36 & \\
\hline No. of tumors & 241 & & & 0.455 \\
\hline Solitary & 189 & 129 & 60 & \\
\hline Multiple & 52 & 40 & 12 & \\
\hline Tumor size $(\mathrm{cm})$ & 241 & & & $0.033^{\mathrm{a}}$ \\
\hline$\leq 5$ & 153 & 100 & 53 & \\
\hline$>5$ & 88 & 69 & 19 & \\
\hline BCLC staging & 217 & & & $0.031^{\mathrm{a}}$ \\
\hline A & 152 & 102 & 50 & \\
\hline$B+C$ & 65 & 53 & 12 & \\
\hline TNM staging & 225 & & & 0.127 \\
\hline I & 96 & 60 & 36 & \\
\hline $\mathrm{II}+\mathrm{III}$ & 129 & 93 & 36 & \\
\hline AFP level (ng/ml) & 237 & & & $0.002^{\mathrm{a}}$ \\
\hline$\leq 300$ & 128 & 78 & 50 & \\
\hline$>300$ & 109 & 87 & 22 & \\
\hline Cirrhosis & 241 & & & 0.225 \\
\hline Yes & 222 & 158 & 64 & \\
\hline No & 19 & 11 & 8 & \\
\hline Metastasis risk & 241 & & & $0.001^{\mathrm{a}}$ \\
\hline Low & 121 & 73 & 48 & \\
\hline High & 120 & 96 & 24 & \\
\hline Relapse & 241 & & & 0.303 \\
\hline Yes & 136 & 99 & 37 & \\
\hline No & 105 & 70 & 35 & \\
\hline
\end{tabular}

avalue indicates a statistically significant difference. MED15, mediator complex subunit 15; AFP, $\alpha$-fetoprotein; BCLC, Barcelona Clinic Liver Cancer.

12.664-661.334; $\mathrm{P}<0.001)$ were independent prognostic factors for OS rate of patients with HCC. These preliminarily results indicate that MED15 may have potential clinical value as a predictive biomarker for OS in patients with $\mathrm{HCC}$, but further studies are needed to reach a clear conclusion.

MED15 expression is positively associated with HIF-1 $\alpha$ in $H C C$. The potential prognostic value of MED15 overexpression prompted the investigation of the mechanism that underlies its upregulation. GSEA was conducted on the MED15 overexpression group. The results revealed that Gene Ontology terms, including 'HYPOXIA' and 'HIF1A and FOXA2', were significantly enriched in MED15 overexpression group $(\mathrm{P}<0.01)$, which indicated that MED15 was regulated by HIF-1 $\alpha$ (Fig. 5A). Furthermore, a positive correlation between HIF-1 $\alpha$ expression and MED15 expression was determined via analysis of the TCGA-LIHC and GSE14520 datasets ( $\mathrm{P}<0.01$; Fig. 5B); therefore, it is hypothesized that MED15 may be regulated by HIF-1 $\alpha$ in HCC.

\section{Discussion}

The present study reported that MED15 is overexpressed in HCC tissues and is closely associated with the neoplasm grade, tumor size, BCLC stage and metastasis risk of patients with HCC. In addition, patients with high MED15 expression 
Table III. Univariate and multivariate analyses (Cox regression analysis) indicating the association between overall survival and the characteristics of hepatocellular carcinoma patients in The Cancer Genome Atlas-Liver Hepatocellular Carcinoma cohort.

\begin{tabular}{|c|c|c|c|c|c|c|}
\hline \multirow[t]{2}{*}{ Variable } & \multicolumn{3}{|c|}{ Univariate analysis } & \multicolumn{3}{|c|}{ Multivariate analysis } \\
\hline & HR & $95 \% \mathrm{CI}$ & P-value & HR & $95 \% \mathrm{CI}$ & P-value \\
\hline Sex & 0.754 & $0.510-1.114$ & 0.156 & & & \\
\hline Age (years) & 0.943 & $0.574-1.552$ & 0.819 & & & \\
\hline Family cancer history & 1.298 & $0.859-1.961$ & 0.216 & & & \\
\hline Lymphocyte infiltration & 1.107 & $0.770-1.592$ & 0.582 & & & \\
\hline Vascular tumor cell invasion & 1.016 & $0.703-1.469$ & 0.932 & & & \\
\hline Neoplasm grade & 1.310 & $0.883-1.945$ & 0.180 & & & \\
\hline Pathologic stage & 1.420 & $0.922-2.187$ & 0.112 & & & \\
\hline AFP level (ng/ml) & 1.566 & $0.976-2.512$ & 0.063 & & & \\
\hline Child-Pugh classification grade & 1.548 & $0.760-3.151$ & 0.229 & & & \\
\hline Liver fibrosis Ishak score & 1.059 & $0.591-1.898$ & 0.846 & & & \\
\hline Relapse & 1.052 & $0.711-1.556$ & 0.801 & & & \\
\hline MED15 expression & 1.581 & $1.039-2.404$ & $0.032^{\mathrm{a}}$ & 1.762 & $1.077-2.882$ & $0.024^{\mathrm{a}}$ \\
\hline
\end{tabular}

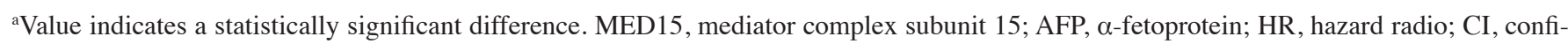
dence interval.
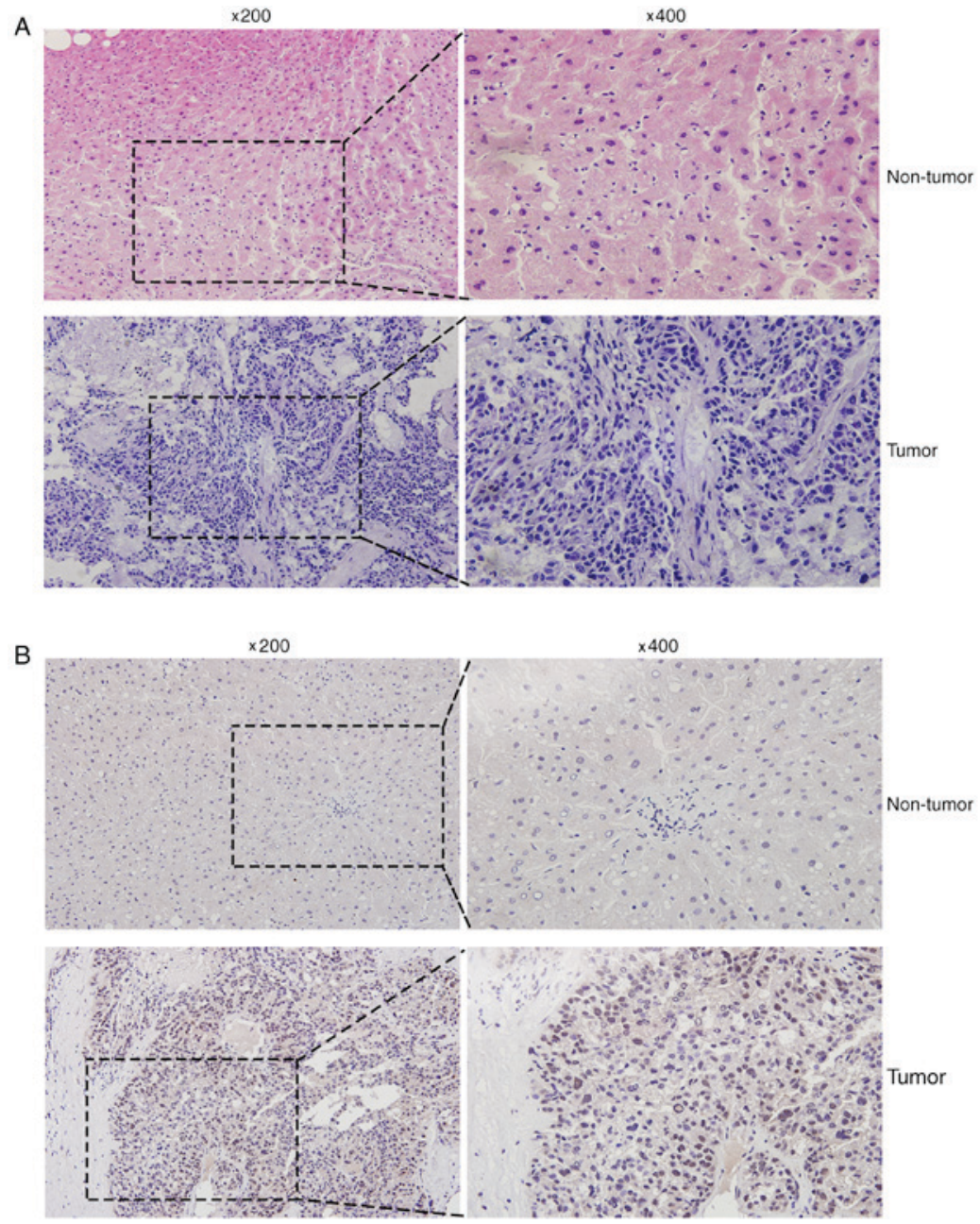

Figure 2. MED15 protein overexpression in HCC specimens. (A) Hematoxylin and eosin staining of non-tumor liver and HCC specimens. (B) Immunohistochemical staining of non-tumor liver and HCC specimens. High MED15 protein expression was observed in HCC specimens, while adjacent non-tumor specimen presented negative or weak expression. MED15 expression appeared as granular brown-colored staining in the nuclei of tumor cells. Representative images with original magnification at x200 and x400 are shown. MED15, mediator complex subunit 15; HCC, hepatocellular carcinoma. 
Table IV. Univariate and multivariate analyses (Cox regression analysis) indicating the association between overall survival and the characteristics of hepatocellular carcinoma patients in the GSE14520 cohort.

\begin{tabular}{|c|c|c|c|c|c|c|}
\hline \multirow[b]{2}{*}{ Variable } & \multicolumn{3}{|c|}{ Univariate analysis } & \multicolumn{3}{|c|}{ Multivariate analysis } \\
\hline & HR & $95 \% \mathrm{CI}$ & P-value & HR & $95 \% \mathrm{CI}$ & P-value \\
\hline Sex & 1.870 & $0.907-3.858$ & 0.090 & & & \\
\hline Age (years) & 0.911 & $0.610-1.360$ & 0.647 & & & \\
\hline No. of tumors & 1.642 & $1.057-2.551$ & $0.027^{\mathrm{a}}$ & 0.389 & $0.203-0.745$ & $0.004^{\mathrm{a}}$ \\
\hline Tumor size $(\mathrm{cm})$ & 1.954 & $1.306-2.924$ & $0.001^{\mathrm{a}}$ & & & 0.493 \\
\hline BCLC staging & 3.357 & $2.211-5.096$ & $<0.001^{\mathrm{a}}$ & 3.615 & $1.982-6.593$ & $<0.001^{\mathrm{a}}$ \\
\hline TNM staging & 3.084 & $1.883-5.053$ & $<0.001^{\mathrm{a}}$ & & & 0.337 \\
\hline AFP level (ng/ml) & 1.710 & $1.141-2.562$ & $0.009^{\mathrm{a}}$ & & & 0.589 \\
\hline Cirrhosis & 5.126 & $1.263-20.804$ & $0.022^{\mathrm{a}}$ & 4.456 & $1.081-18.367$ & 0.039 \\
\hline Metastasis risk & 2.281 & $1.504-3.459$ & $<0.001^{\mathrm{a}}$ & 2.41 & $1.523-3.814$ & $0.001^{\mathrm{a}}$ \\
\hline Relapse & 122.187 & $17.008-877.810$ & $<0.001^{\mathrm{a}}$ & 91.516 & $12.664-661.334$ & $<0.001^{\mathrm{a}}$ \\
\hline MED15 expression & 1.517 & $0.950-2.425$ & 0.081 & & & \\
\hline
\end{tabular}

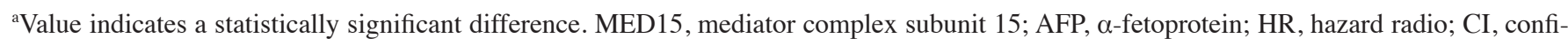
dence interval.

A Human protein atlas MED15

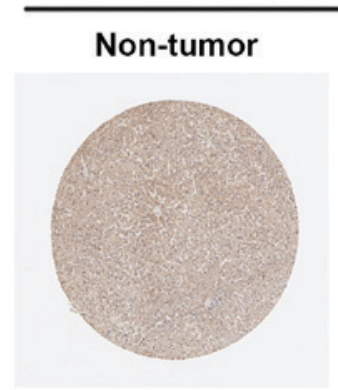

$100 \mathrm{X}$

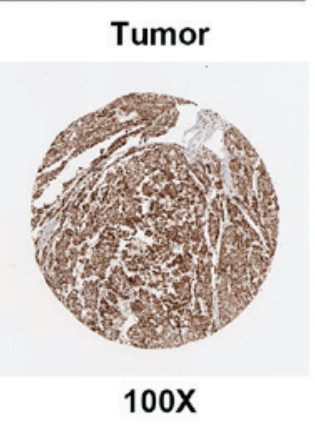

$100 \mathrm{X}$
B

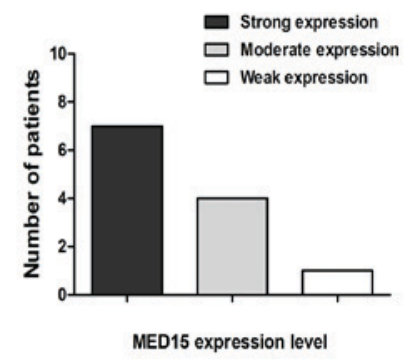

C

MED15 Expression level

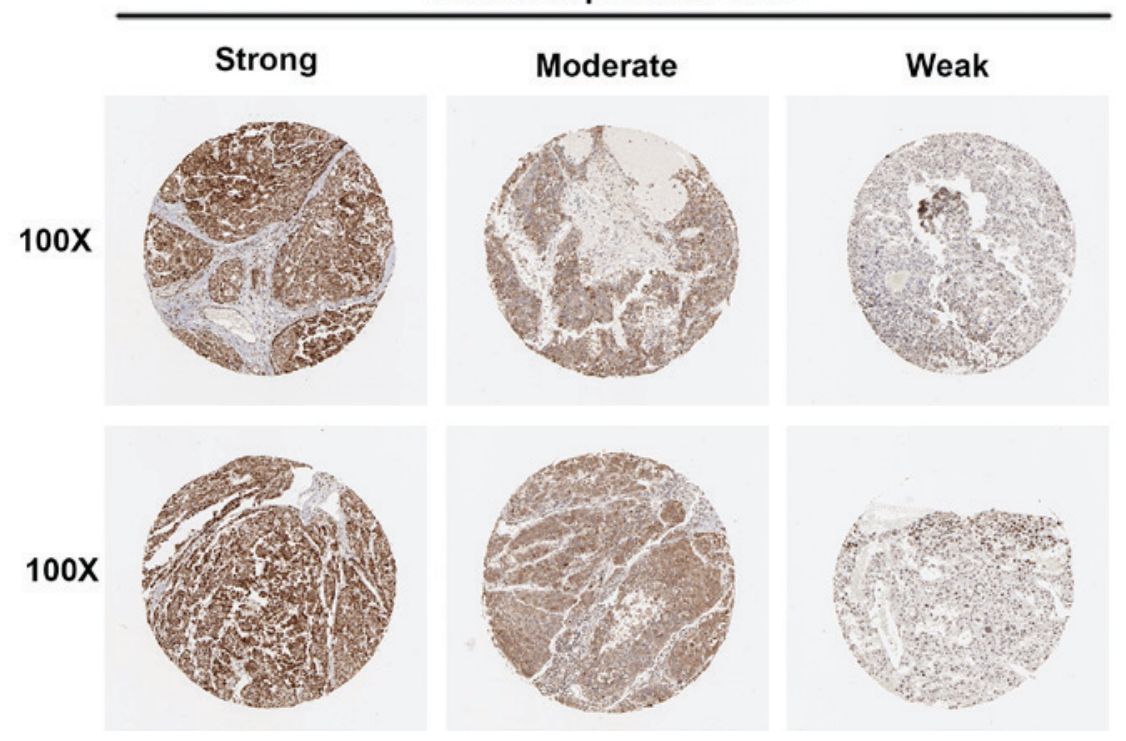

Figure 3. MED15 protein expression is elevated in HCC samples included in the Human Protein Atlas. (A) MED15 expression in normal liver and HCC tissues obtained from the Human Protein Atlas. (B) Proportion of patients with different MED15 immunostaining intensity in HCC specimens and (C) representative images of immunostaining. Images at magnification x 100 were obtained from the Human Protein Atlas online database. MED15, mediator complex subunit 15; HCC, hepatocellular carcinoma. 
A

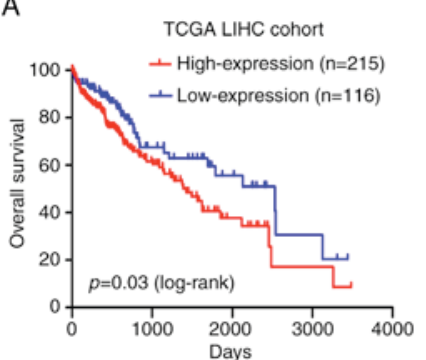

$\mathrm{B}$

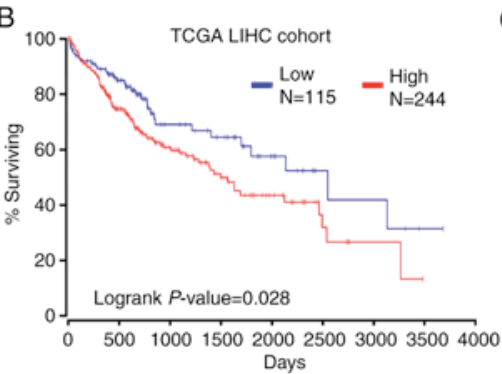

C

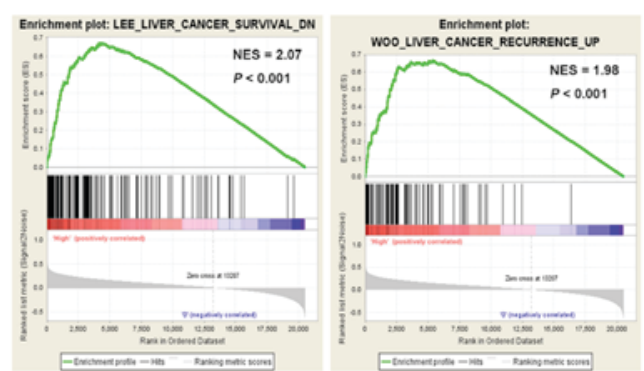

D

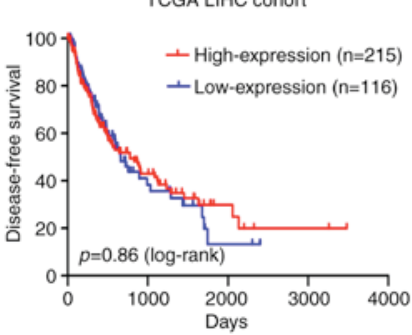

E

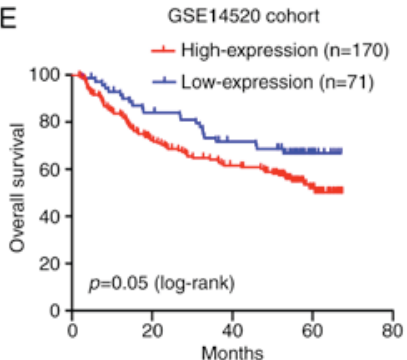

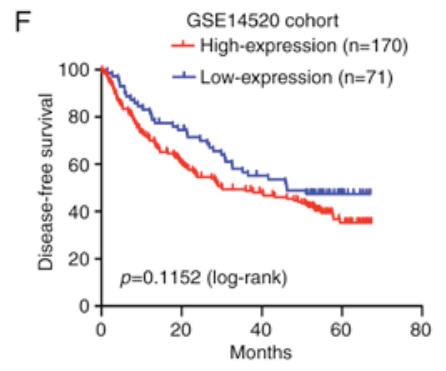

Figure 4. Correlation between MED15 expression and survival of patients with HCC in the TCGA-LIHC and GSE14520 cohorts. (A) Reduced OS time was detected for patients with high MED15 expression compared with those with low MED15 expression in the TCGA-LIHC cohort. (B) Kaplan-Meier survival analysis of OS rate from the TCGA-LIHC dataset from the OncoLnc online system. (C) Gene Set Enrichment Analysis results revealed that MED15 overexpression was correlated with a low survival rate and high recurrence rate. (D) DFS time exhibits no difference between patients with high or low MED15 expression in the TCGA-LIHC cohort. (E) OS and (F) DFS rates in the GSE14520 cohort were assessed by Kaplan-Meier survival analysis. MED15, mediator complex subunit 15; HCC, hepatocellular carcinoma; TCGA-LIHC, The Cancer Genome Atlas-Liver Hepatocellular Carcinoma; OS, overall survival; DFS, disease-free survival.

A

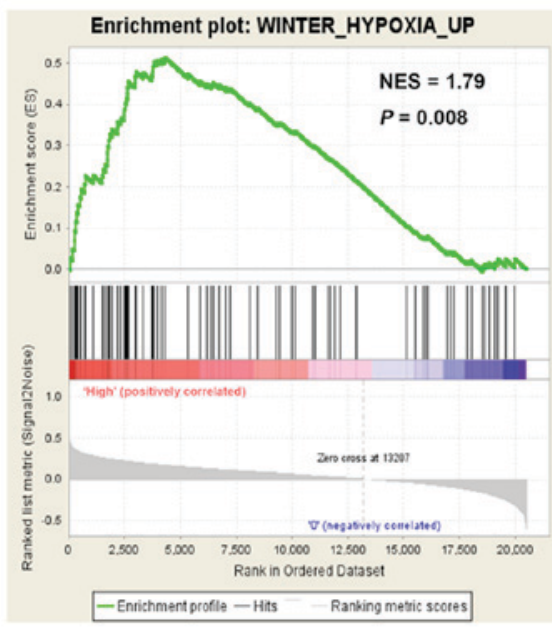

B

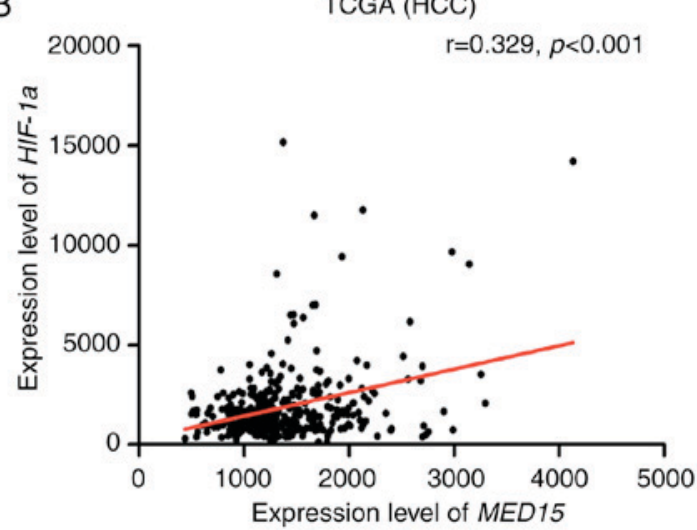

Enrichment plot:

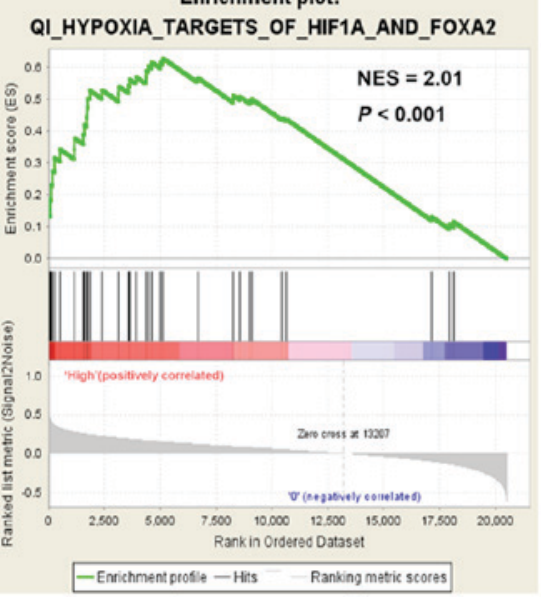

GSE14520

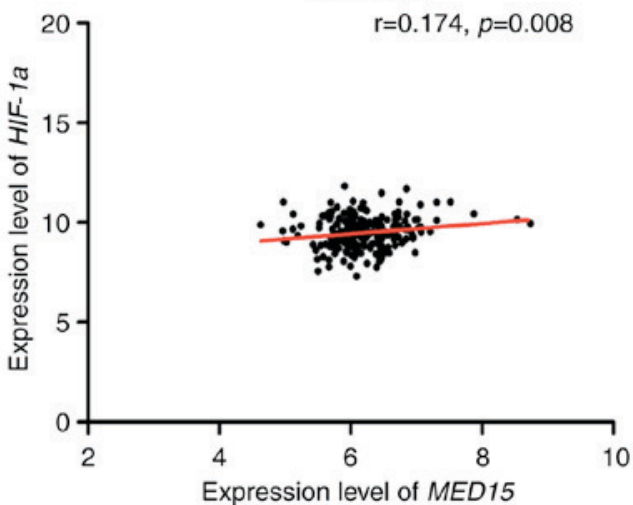

Figure 5. Correlation between MED15 expression and HIF-1 $\alpha$ expression. (A) Gene set enrichment analysis revealed that MED15 overexpression was associated with hypoxia and HIF-1 $\alpha$ targets. (B) MED15 expression was positively associated with HIF-1 $\alpha$ expression in the TCGA-LIHC and GSE14520 cohorts. MED15, mediator complex subunit 15; TCGA-LIHC, The Cancer Genome Atlas-Liver Hepatocellular Carcinoma; HIF-1 $\alpha$, hypoxia-inducible factor 1 $\alpha$. 
levels exhibited a poor disease prognosis. These observations indicated that MED15 serves a role in liver tumorigenesis and may be a potential prognostic factor for HCC.

In the present study, MED15 was observed to be upregulated in human HCC specimens when compared with its expression in the corresponding adjacent non-tumor specimens. It has been reported that MED15 is dysregulated in several malignancies, including breast cancer, prostate cancer and testicular germ cell tumors (6,11-13). For instance, MED15 is overexpressed in tissues obtained from primary tumors, metastasizing lymph nodes and recurrent tumors, when compared with benign tissues in HNSCC (10). Meanwhile, a previous study demonstrated that the methylation levels of the MED15 promoter were significantly elevated in DNA samples from HNSCC tissues compared with those in adjacent normal tissues. Notably, hypermethylation of the MED15 promoter in HNSCC tissues appeared to promote malignant transformation instead of functioning as a typical tumor suppressor gene (16). These findings further confirm the oncogenic potential of MED15 in HNSCC. To date, research into the role of MED15 in cancer consistently reported its overexpression in tumor tissues and its oncogenic functions in regulating the initiation and progression of several malignancies. Furthermore, consistent with the localization of MED15 in testicular germ cell tumor tissues, prostate cancer tissues and HNSCC tissues $(10,11,13)$, the present study observed that MED15 was primarily located in the nucleus of cells in HCC tissues.

The analysis conducted in the current study revealed a significant association between MED15 overexpression and a poor clinical outcome. Accumulating evidence has suggested that MED15 overexpression is a predictor of poor prognosis for several types of human cancer, including prostate cancer, HNSCC and breast cancer $(6,10,11,16)$. Shaikhibrahim et al (11) had identified MED15 nuclear overexpression to be associated with poor prognosis in castration-resistant prostate cancer. Furthermore, it has been reported that MED15 overexpression correlates with a high mortality rate in patients with HNSCC (10). In the current study, these findings were extended to liver cancer, since MED15 overexpression was observed to be indicative of a highly lethal phenotype in HCC. The analysis of clinical data from patients with HCC patients in the TCGA-LIHC cohort further suggested that MED15 expression may be an independent prognostic survival indicator. However, similar results were not observed in the GSE14520 cohort analysis in the present study, possibly owing to inconsistent enrollment of patients at different research centers. The number of cases may also be important in the accuracy of these results, since the number of patients included in the TCGA-LIHC cohort was larger in comparison with that in the GSE14520 cohort. Nonetheless, the present study proposes that MED15 may be regarded as a novel indicator for identifying patients with a poor prognosis in HCC.

Previous studies demonstrated that certain transcription factors regulate and control the expression of specific genes (17-20). For instance, HMG box-containing protein 1 has been reported to participate in Ras-induced premature senescence by regulating $\mathrm{p} 16^{\mathrm{INK} 4 \mathrm{~A}}$ expression (21). In addition, HIF-1 $\alpha$ regulates the expression of genes encoding proteins with key roles in cancer biology (22), which can directly activate vascular endothelial growth factor (VEGF) and VEGF receptor 1 transcription via hypoxia response element binding (23). In the current study, GSEA was used to explore pathways that may be responsible for high MED15 expression levels, and HIF-1 $\alpha$ was identified as one of the potential regulators. HIF- $1 \alpha$ is known to be an important transcription factor in hypoxia response and to function in the development and progression of tumors, including liver cancer (24-26). The results of the present study demonstrated that the expression levels of MED15 in the TCGA-LIHC and GSE14520 datasets were positively associated with HIF-1 $\alpha$ expression, suggesting that MED15 may be a target of HIF-1 $\alpha$.

In conclusion, the present study reported that MED15 was upregulated in HCC tissues and that high MED15 expression was correlated with poor prognosis in patients with HCC. To the best of our knowledge, it was revealed for the first time that an association exists between MED15 and human liver cancer, providing valuable information for further studies on HCC. However, detailed investigations are required to better understand the roles of MED15 in liver tumorigenesis and cancer progression.

\section{Acknowledgements}

The authors would like to thank Professor Xin Wei Wang for sharing information regarding the GSE14520 dataset online. The current study was supported by the National Nature Science Foundation of China (grant nos. 81372283, 81472711, 81401180, 81672756 and 91540111), the Guangdong Province Universities and Colleges Pearl River Scholar Funded Scheme (grant no. 2015), and the Natural Science Foundation of Guangdong Province (grant no. 2014A030311013).

\section{References}

1. Sia D, Villanueva A, Friedman SL and Llovet JM: Liver cancer cell of origin, molecular class, and effects on patient prognosis. Gastroenterology 152: 745-761, 2017.

2. Torre LA, Siegel RL, Ward EM and Jemal A: Global cancer incidence and mortality rates and trends-An update. Cancer Epidemiol Biomarkers Prev 25: 16-27, 2016.

3. Llovet JM, Zucman-Rossi J, Pikarsky E, Sangro B, Schwartz M, Sherman M and Gores G: Hepatocellular carcinoma. Nat Rev Dis Primers 2: 16018, 2016.

4. Kato Y, Habas R, Katsuyama Y, Näär AM and He X: A component of the ARC/Mediator complex required for TGF beta/Nodal signalling. Nature 418: 641-646, 2002.

5. Yang F, Vought BW, Satterlee JS, Walker AK, Jim Sun ZY, Watts JL, DeBeaumont R, Saito RM, Hyberts SG, Yang S, et al: An ARC/Mediator subunit required for SREBP control of cholesterol and lipid homeostasis. Nature 442: 700-704, 2006.

6. Zhao M, Yang X, Fu Y, Wang H, Ning Y, Yan J, Chen YG and Wang G: Mediator MED15 modulates transforming growth factor beta (TGF $\beta) /$ Smad signaling and breast cancer cell metastasis. J Mol Cell Biol 5: 57-60, 2013.

7. Yang X and Yang F: Mediating lipid biosynthesis: Implications for cardiovascular disease. Trends Cardiovasc Med 23: 269-273, 2013.

8. Nakatsubo T, Nishitani S, Kikuchi Y, Iida S, Yamada K, Tanaka A and Ohkuma Y: Human mediator subunit MED15 promotes transcriptional activation. Drug Discov Ther 8: 212-217, 2014.

9. Berti L, Mittler G, Przemeck GK, Stelzer G, Günzler B, Amati F, Conti E, Dallapiccola B, Hrabé de Angelis M, Novelli G and Meisterernst M: Isolation and characterization of a novel gene from the DiGeorge chromosomal region that encodes for a mediator subunit. Genomics 74: 320-332, 2001.

10. Shaikhibrahim Z, Offermann A, Halbach R, Vogel W, Braun M, Kristiansen G, Bootz F, Wenzel J, Mikut R, Lengerke C, et al: Clinical and molecular implications of MED15 in head and neck squamous cell carcinoma. Am J Pathol 185: 1114-1122, 2015. 
11. Shaikhibrahim Z, Menon R, Braun M, Offermann A, Queisser A, Boehm D, Vogel W, Rüenauver K, Ruiz C, Zellweger T, et al: MED15, encoding a subunit of the mediator complex, is overexpressed at high frequency in castration-resistant prostate cancer. Int J Cancer 135: 19-26, 2014.

12. Offermann A, Vlasic I, Syring I, Vogel W, Ruiz C, Zellweger T, Rentsch CA, Hagedorn S, Behrends J, Nowak M, et al: MED15 overexpression in prostate cancer arises during androgen deprivation therapy via PI3K/mTOR signaling. Oncotarget 8: 7964-7976, 2017.

13. Klümper N, Syring I, Offermann A, Shaikhibrahim Z, Vogel W, Müller SC, Ellinger J, Strauß A, Radzun HJ, Ströbel P, et al: Differential expression of Mediator complex subunit MED15 in testicular germ cell tumors. Diagn Pathol 10: 165, 2015.

14. Schiano C, Casamassimi A, Rienzo M, de Nigris F, Sommese L and Napoli C: Involvement of Mediator complex in malignancy. Biochim Biophys Acta 1845: 66-83, 2014.

15. Livak KJ and Schmittgen TD: Analysis of relative gene expression data using real-time quantitative PCR and the 2(-Delta Delta $\mathrm{C}(\mathrm{T})$ ) method. Methods 25: 402-408, 2001.

16. Ovchinnikov DA, Wan Y, Coman WB, Pandit P, Cooper-White JJ, Herman JG and Punyadeera C: DNA methylation at the novel $\mathrm{CpG}$ sites in the promoter of MED15/PCQAP gene as a biomarker for head and neck cancers. Biomarker Insights 9: 53-60, 2014.

17. Gañán-Gómez I, Wei Y, Yang H, Boyano-Adánez MC and García-Manero G: Oncogenic functions of the transcription factor Nrf2. Free Radic Biol Med 65: 750-764, 2013.

18. Luo D, Wang Z, Wu J, Jiang C and Wu J: The role of hypoxia inducible factor-1 in hepatocellular carcinoma. Biomed Res Int 2014: 409272, 2014.
19. Monga SP: $\beta$-catenin signaling and roles in liver homeostasis, injury, and tumorigenesis. Gastroenterology 148: 1294-1310, 2015.

20. Rogacki K, Kasprzak A and Stępiński A: Alterations of Wnt/ $\beta$-catenin signaling pathway in hepatocellular carcinomas associated with hepatitis C virus. Pol J Pathol 1: 9-21, 2015.

21. Li H, Wang W, Liu X, Paulson KE, Yee AS and Zhang X: Transcriptional factor HBP1 targets P16(INK4A), upregulating its expression and consequently is involved in Ras-induced premature senescence. Oncogene 29: 5083-5094, 2010.

22. Semenza GL: Defining the role of hypoxia-inducible factor 1 in cancer biology and therapeutics. Oncogene 29: 625-634, 2010.

23. Liao D and Johnson RS: Hypoxia: A key regulator of angiogenesis in cancer. Cancer Metastasis Rev 26: 281-290, 2007.

24. Wu XZ, Xie GR and Chen D: Hypoxia and hepatocellular carcinoma: The therapeutic target for hepatocellular carcinoma. J Gastroenterol Hepatol 22: 1178-1182, 2007.

25. Xiang ZL, Zeng ZC, Fan J, Tang ZY, He J, Zeng HY and Chang JY: The expression of HIF-1 $\alpha$ in primary hepatocellular carcinoma and its correlation with radiotherapy response and clinical outcome. Mol Biol Rep 39: 2021-2029, 2012.

26. Lin D and Wu J: Hypoxia inducible factor in hepatocellular carcinoma: A therapeutic target. World J Gastroenterol 21: 12171-12178, 2015

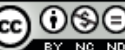

This work is licensed under a Creative Commons Attribution-NonCommercial-NoDerivatives 4.0 International (CC BY-NC-ND 4.0) License. 